\title{
Pattern Selection and Super-patterns in the Bounded Confidence Model
}

\author{
E. Ben-Naim ${ }^{1}$ and A. Scheel ${ }^{2}$ \\ ${ }^{1}$ Theoretical Division and Center for Nonlinear Studies, \\ Los Alamos National Laboratory, Los Alamos, New Mexico 87545 USA \\ ${ }^{2}$ School of Mathematics, University of Minnesota, Minneapolis, MN 55455, USA
}

\begin{abstract}
We study pattern formation in the bounded confidence model of opinion dynamics. In this random process, opinion is quantified by a single variable. Two agents may interact and reach a fair compromise, but only if their difference of opinion falls below a fixed threshold. Starting from a uniform distribution of opinions with compact support, a traveling wave forms and it propagates from the domain boundary into the unstable uniform state. Consequently, the system reaches a steady state with isolated clusters that are separated by distance larger than the interaction range. These clusters form a quasi-periodic pattern where the sizes of the clusters and the separations between them are nearly constant. We obtain analytically the average separation between clusters $L$. Interestingly, there are also very small quasi-periodic modulations in the size of the clusters. The spatial periods of these modulations are a series of integers that follow from the continued fraction representation of the irrational average separation $L$.
\end{abstract}

PACS numbers: 89.75.Kd, 82.40.Ck, 05.45.-a

The so-called "bounded-confidence" model [1-7] and variants thereof have been widely used to model opinion dynamics [8 14] and have attracted a considerable amount of interest. The bounded confidence model is appealing because it captures the tendency for reaching compromise through social interactions, while also taking into account a certain degree of conviction. Numerical studies show that political parties emerge in the bounded confidence model as a result of a pattern formation process [1, 3, 4]. In this letter, we obtain analytically the wavelength governing this process.

We focus on a version of the bounded confidence model in which opinions are quantified as discrete variables $1 \leq n \leq N$. In each interaction, two agents with opinions $n_{1}$ and $n_{2}$ change their initial opinions by adopting the average opinion $\left(n_{1}, n_{2}\right) \rightarrow\left(\frac{n_{1}+n_{2}}{2}, \frac{n_{1}+n_{2}}{2}\right)$; such a compromise occurs only when the opinion difference is smaller than some fixed threshold $\left|n_{1}-n_{2}\right| \leq \sigma$. We set the threshold $\sigma=2$ and exclude interactions between agents whose opinion difference equals one, $\left|n_{1}-n_{2}\right|=1$, to ensure that opinions remain discrete variables. In this simplified version of the bounded confidence model, opinions change according to [4]

$$
(n-1, n+1) \rightarrow(n, n) .
$$

Clearly, this process conserves population and opinion.

Let $P_{n}(t)$ be the probability density of agents with opinion $n$ at time $t$. This density obeys the rate equation

$$
\frac{d P_{n}}{d t}=2 P_{n-1} P_{n+1}-P_{n}\left(P_{n-2}+P_{n+2}\right) .
$$

In writing this equation, we implicitly take the infinite population limit. It is simple to check that Eq. (2) conserves population, $\sum_{n} P_{n}$, and opinion, $\sum_{n} n P_{n}$.

The initial distribution of opinions is uniform with compact support,

$$
P_{n}(0)= \begin{cases}0 & n<1 \\ 1 & 1 \leq n \leq N \\ 0 & N<n .\end{cases}
$$

We view the parameter $N$ as the opinion "spectrum," and also note that $N$ is the only parameter in the model. The evolution equation (2) is invariant under the scaling transformation $P \rightarrow \alpha P$ and $t \rightarrow t / \alpha$ and hence, we may set the uniform initial density to unity. This choice allows us to compare systems with different opinion spectrums.

The nature of the interaction (11), also reflected by the evolution equation (2), implies that any probability density that satisfies $P_{n-1} P_{n+1}=0$ for all $n$ is stationary. Clearly, there are infinitely many such steady-state solutions. Starting from the (unstable) initial condition (3), the deterministic rate equation (2) evolves the system toward one of those (stable) steady-state solutions 3 .

In the final state (i.e., in the limit $t \rightarrow \infty$ ), the system reaches a steady state where $P_{n-1}(\infty) P_{n+1}(\infty)=0$ for all $n$. In this state, there are multiple opinion "clusters" with each cluster localized to two neighboring lattice sites (see figure1). These clusters are noninteracting because the separation between them exceeds the interaction range.

To quantify the size and opinion of each cluster, we compute for every pair of occupied lattice sites the mass $m=P_{n}(\infty)+P_{n+1}(\infty)$ and the non-integer position $x=\left[n P_{n}(\infty)+(n+1) P_{n+1}(\infty)\right] / m$. Let $m_{i}$ be the mass of the $i$ th cluster and $x_{i}$ be the position of the $i$ th cluster. Conservation of population and opinion sets the sum rules $\sum_{i} m_{i}=N$ and $\sum_{i} m_{i} x_{i}=N(N+1) / 2$.

Unlike the probability density, the cluster mass forms a quasi-periodic pattern (see figure 1) as clusters of nearlyidentical mass are separated by a nearly-identical distance. This pattern can be characterized by the average 

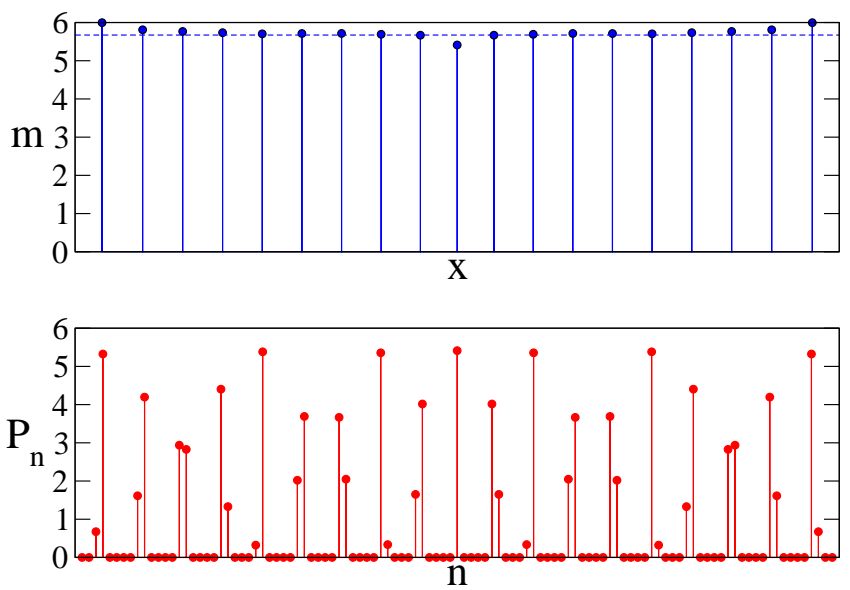

FIG. 1: [bottom] The probability density $P_{n}(\infty)$ versus $n$. [top] The cluster mass $m$ versus position $x$. . Shown are results of integration of (2)-(3) with $N=109$. The dashed line corresponds to the theoretical value $L=5.671820$

separation $L$ between clusters.

$$
L=\lim _{N \rightarrow \infty}\langle m\rangle .
$$

With this definition, the average number of clusters scales as $N / L$ in the limit $N \rightarrow \infty$. Previous numerical studies reported the value $L \approx 5.67$ [4]. In this letter, we use theoretical methods to analyze the evolution of the probability density $P_{n}$ and analytically obtain $L$ as the wavelength that governs the underlying pattern formation process.

The uniform initial state (3) is unstable with respect to perturbations that propagate from either boundary into the unstable uniform state [15, 16]. By substituting the small periodic disturbance

$$
P_{n}(t)-1 \propto \exp [i(k n-\omega t)]
$$

into the evolution equation (2) we find the dispersion relation between frequency $\omega$ and wavelength $k$,

$$
\omega=2 i(2 \cos k-\cos 2 k-1) .
$$

Because the quantity $-i \omega$ is positive for $0<k<\pi / 2$, perturbations with wavenumber in that range initially grow exponentially with time. The fastest growing mode, by ordinary linear stability analysis, follows immediately from (6). The maximum of $-i \omega$ in (6) is set by $d \omega / d k=0$ which yields $k_{\text {linear }}=\pi / 3$ or alternatively $L_{\text {linear }}=2 \pi / k_{\text {linear }}$, that is, $L_{\text {linear }}=6$.

The perturbations propagate from the stable state into the unstable state at a constant velocity $v$ (see figure 2). A saddle point analysis shows that the propagation velocity $v$ obeys (for a comprehensive review see [17] and also [18-21])

$$
v=\frac{d \omega}{d k}=\frac{\operatorname{Im}[w]}{\operatorname{Im}[k]} .
$$

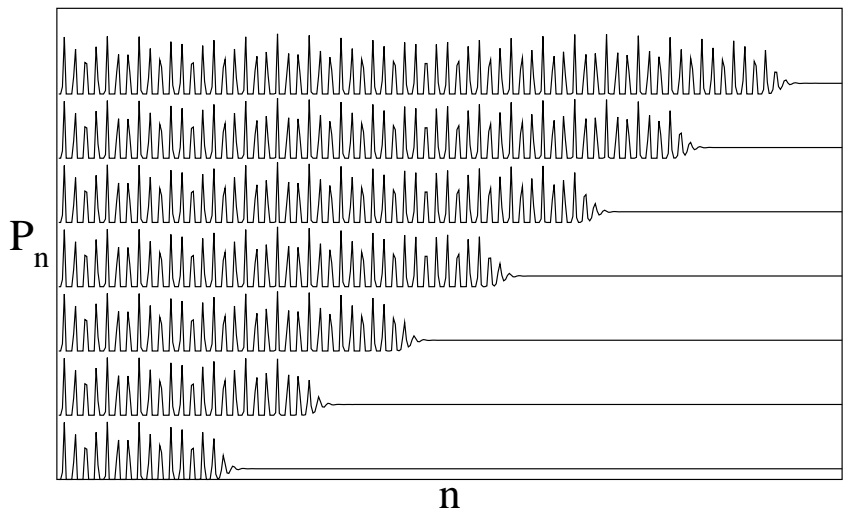

FIG. 2: Propagation from the stable into the unstable state. Shown is $P_{n}(t)$ at equally spaced time intervals versus $n$. The curves $P_{n}(t)$ are shifted vertically with the earliest time at the bottom and the latest time on top.

The solution to this equation is the complex wavenumber $k_{*} \equiv k_{\text {front }}+i \lambda$ with $k_{\text {front }}=1.183032$ [4]. The constant $\lambda=0.467227$ characterizes the exponential decay of these periodic perturbations $P_{n}-1 \sim e^{-\lambda(n-v t)} e^{i k_{\text {front }}(n-v t)}$. The wavelength of perturbations at the leading edge of the front is $L_{\text {front }}=2 \pi / k_{\text {front }}$ or explicitly,

$$
L_{\text {front }}=5.311086 \text {. }
$$

Moreover, the propagation velocity $v=\operatorname{Im}\left[w_{*}\right] / \operatorname{Im}\left[k_{*}\right]$ where $w_{*} \equiv w\left(k_{*}\right)$ is

$$
v=3.807397 .
$$

Our numerical results confirm that in the leading edge of the propagating front, the wavelength of the periodic deviations from the uniform state is indeed given by (8).

Far behind the traveling wave, that is, in the wake of the wave, the system reaches a steady state with $P_{n-1} P_{n+1}=0$ for all $n \ll v t$. In this region, clusters are fully-developed. Interestingly, far behind the propagating front, the pattern that forms, and which ultimately controls the spacing between clusters, has a larger wavelength due to a Doppler-like effect. The frequency of oscillations in the front $w_{*}-k_{*} v$, measured in the comoving frame, translates to a zero frequency in the rest frame and hence, to stationary patterns, precisely for the wavenumber [17, 20, 22]

$$
k=k_{*}-\frac{w_{*}}{v} .
$$

We note that unlike the Doppler effect, the normalized shift in wavenumber $\left(k_{*}-k\right) / k_{*}$ does not equal a ratio of two velocities as it is complex. The resulting wavenumber (10) is $k=1.107789$ and the corresponding wavelength $L=2 \pi / k$ is

$$
L=5.6718200283 .
$$




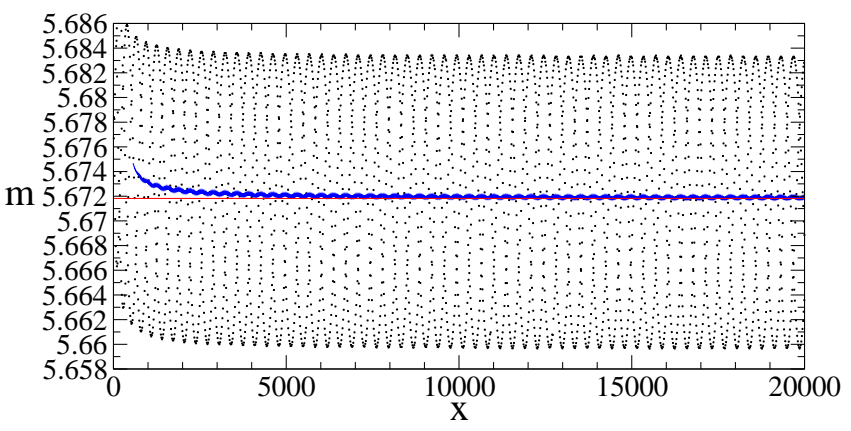

FIG. 3: The cluster mass $m$ versus position $x$. The red line corresponds to the theoretical value (11) and the blue line, to a 100-point running average.

Hence, out of the entire range of possible wavelengths corresponding to linearly unstable perturbations, $0<L<6$, the wavenumber (11) is "selected" by the dynamics of Eq. (2). We also note the inequality $L_{\text {front }}<L<L_{\text {linear }}$. Our numerical results give excellent confirmation of the theoretical prediction (see Fig. 31): the numericallymeasured wavelength $L=5.67185$ is within $10^{-5}$ of (11).

To efficiently perform the computation, we integrated the equations using a lattice with fixed size $\mathcal{N}$ that is moving at the same speed as the traveling wave. $\mathrm{Nu}$ merical integration in this co-moving reference frame is feasible because far ahead of the traveling wave $P_{n}=1$ and far behind it, the system settles into a steady-state. The lattice is shifted by one lattice site, $n \rightarrow n+1$ whenever the deviation from the uniform state at the extreme lattice site, far ahead of the traveling front, exceeds an infinitesimal threshold, $\left|P_{\mathcal{N}}-1\right|>\epsilon$. We used a RungeKutta $(4,5)$ integration method with adaptive step size below $10^{-3}$ on a lattice of size $\mathcal{N}=2,000$ and the threshold $\epsilon=10^{-250}$, well above the smallest machine precision $10^{-308}$. To resolve the leading edge at this precision, we integrated the equations for $Q_{n}=P_{n}-1$ where the leading edge decays to the constant state $Q_{n}=0$. Whenever the lattice is shifted by one site, time and the probability density at the site $n=200$, well behind the leading edge, were recorded. This approach allows us to integrate the equation to times $t \approx 3 \times 10^{6}$ and effectively, study systems with very large opinion spectrums $N \approx 10^{6}$.

The number of shifts at time $t$ directly measures the front position $x_{f}$. As shown in figure 4 The numericallymeasured propagation velocity $v=3.80732$ is in excellent agreement with the theoretical prediction (9). It is remarkable that our computation, for which the ratio between system size and wavelength is moderate, $\mathcal{N} / L \approx 350$, yields such high-precision measurements of $L$ and $v$. In general, a cutoff error $\epsilon$ in the propagating front results in logarithmic correction $\delta \sim(\ln \epsilon)^{-2}$ in the propagation velocity [23]. Moreover, the cutoff decays exponentially with system size, $\epsilon \sim \exp (-\lambda \mathcal{N})$. By combining these two scaling laws, we find the algebraic

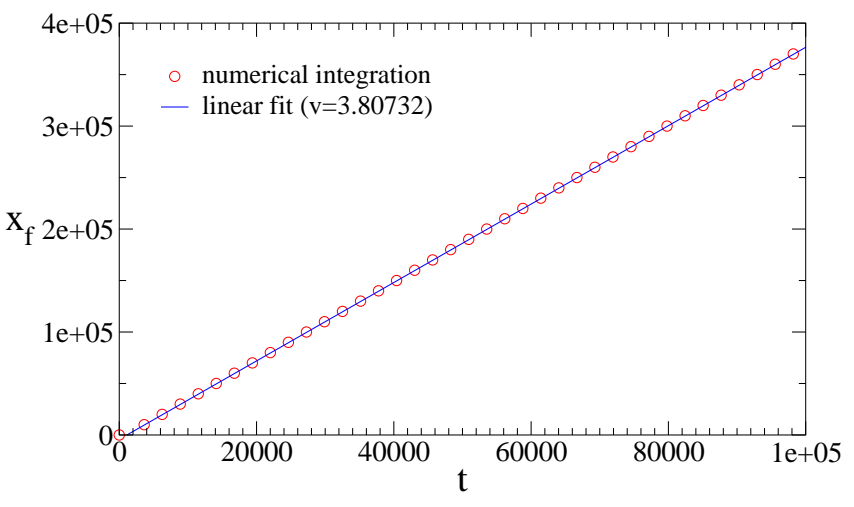

FIG. 4: The front location $x_{f}$ versus time $t$.

relationship between system size $\mathcal{N}$ and correction $\delta$ to the velocity, $\delta \sim(\lambda \mathcal{N})^{-2}$. The velocity correction we observe, $\delta \approx 10^{-5}$ for $\mathcal{N} \approx 10^{3}$, is consistent with this scaling law.

The irrational wavelength $L$ in (11) is not commensurate with the unit lattice spacing. As a result, the patterns are not strictly periodic but rather, they are quasiperiodic (see figure 3). Interestingly, we observed small but striking "super-patterns" induced by near-resonances between $L$ and the lattice spacing. The integer periods of these super-patterns are found from a continuous-fraction expansion of the wavelength

$$
L=5+\frac{1}{1+\frac{1}{2+\frac{1}{21+\frac{1}{4+\ldots}}}} \equiv 6, \frac{17}{3}, \frac{363}{64}, \frac{1469}{259}, \cdots .
$$

Indeed, 3 clusters can be accommodated within the integer period 17 . Figure 2 for the probability density $P_{n}$ shows that a large peak in the quantity $P_{n}$ is usually followed by two smaller ones. Hence, the system exhibits quasi-periodic behavior with integer period 17.

Furthermore, there is also quasi-periodic arrangement of clusters with integer period 363. Figure 5 shows that the cluster mass varies in the range $L-\Delta<m<L+\Delta$. The variation in cluster mass is very small $\Delta / m \approx 2 \times 10^{-3}$. As a function of position, these small variations in cluster mass repeat with integer period 363. According to the continued fraction (12), 64 clusters form this pattern. This intriguing behavior was overlooked in previous studies that used much smaller values of the opinion spectrum $N[3,4]$.

Interestingly, the simple evolution equation (2) leads to a hierarchical patterns, governed by a series of integer periods. The primary pattern, as shown in figure 1 consists of a nearly-periodic arrangement of clusters with nearlyidentical separation. On the first hierarchical level, $M_{1}$ clusters are arranged in a nearly periodic super-pattern with period $L_{1}$. On the second hierarchical level, $M_{2}$ patterns form a more intricate super-pattern with the integer period $L_{2}$. The fractions $M_{1} / L_{1}, M_{2} / L_{2}$, and so 


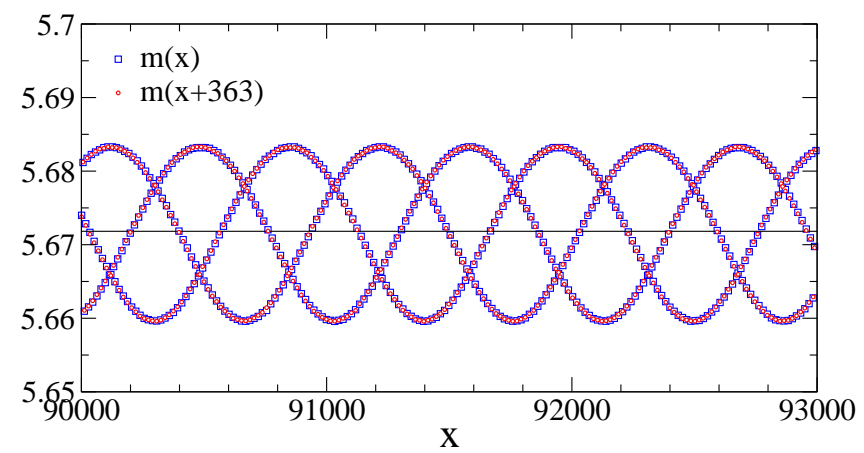

FIG. 5: The cluster mass $m$ versus position $x$ (large square) and versus shifted position $x+363$ (small circle). The line corresponds to the theoretical value (11).

on are rational approximations of the wavelength $L$ that follow from its continued fraction representation (12).

To examine the robustness of the above behavior, we also considered the compromise process $(n-1, n+2) \rightarrow$ $(n, n+1)$ where the interaction range is three lattice sites. In this case, the probability density evolves according to the rate equation

$$
\frac{d P_{n}}{d t}=P_{n-2} P_{n+1}+P_{n-1} P_{n+2}-P_{n}\left(P_{n-3}+P_{n+3}\right) .
$$

This equation conserves population and opinion. Starting from the initial condition (3), the probability density evolves toward a steady state where $P_{n}(\infty) P_{n+3}(\infty)=0$ for all $n$, and therefore, opinion clusters are now localized to three consecutive lattice sites. By substituting (5) into (13), the dispersion relation is

$$
\omega=2 i(\cos k+\cos 2 k-\cos 3 k-1) .
$$

By repeating the analysis leading to (11) we obtain the average separation between clusters and propagation velocity

$$
L=8.5502770500 \text { and } v=2.50631 \text {. }
$$

Also, the wavelength of patterns nucleating at the front is $L_{\text {front }}=8.02282$. Numerical integration of the evolution equation gives $L=8.5503$ and $v=2.5063$, in excellent agreement with the theoretical predictions.

Figure 6] demonstrates the emergence of superpatterns. In this case, continued fraction representation of the wavelength (15) gives the rational approximations

$$
L=8+\frac{1}{1+\frac{1}{1+\frac{1}{4+\frac{1}{2+\ldots}}}} \equiv 9, \frac{17}{2}, \frac{77}{9}, \frac{171}{20}, \frac{1445}{169} \ldots .
$$

Figure [6] shows that modulations in cluster mass are periodic and well-characterized by the integer period 1445 , that follows from the continued fraction (16). Accordingly, the super-pattern consists of 169 clusters. Furthermore, the amplitude of the variations is very small, $\Delta / m \approx 5 \times 10^{-4}$.

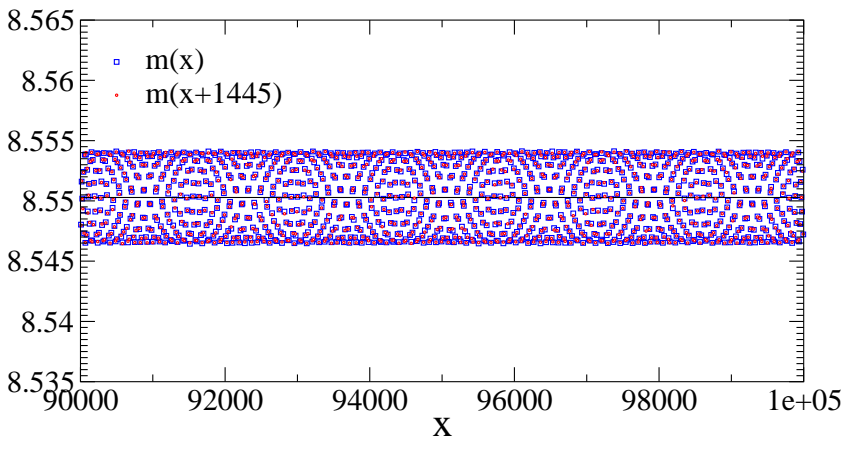

FIG. 6: The cluster mass $m$ versus position $x$ (large square) and versus shifted position $x+1445$ (small circle) for the three-site interaction model in (13). The line corresponds to the theoretical value (15).

We also examined a linear interpolation between (2) and (13), modeling a compromise process where secondor third-neighbor interactions occur with relative weights $\tau$ and $1-\tau$, respectively. We found excellent agreement between the theoretical predictions and the numerical results for the wavelength $L$ and the velocity $v$ for all values of $\tau$. As expected, the wavelength decreases monotonically with the mixing parameter $\tau$, which decreases the effective interaction range. Surprisingly, however, the velocity is not a monotonic function as it reaches a minimum for $\tau \approx 0.88$. Thus, replacing a small fraction of the second with third-neighbor interactions slows down the spreading of the compromise process.

Moreover, we do not observe "mode-locking". In this phenomenon, common in pattern forming systems that are exposed to an external spatially-periodic forcing, there is pronounced locking of the wavelength to that of the forcing [24, 25]. In lattice systems, one therefore expects the observed wavelength to be an integer, in resonance with the unit lattice spacing, whenever the predicted wavelength is close to an integer. In our case, the predicted wavelength $L$ is integer ( 6 or 7 or 8 ) for particular values of the mixing parameter $\tau$. We studied values of $\tau$ near those resonances but did not observe any locking: the wavelength varies smoothly and adheres to the predicted values. Further, we did not observe subharmonic resonances as in 26 29], where the observed wavelength is an integer multiple of the predicted wavelength.

We now briefly discuss the original bounded confidence model introduced by Weisbuch et al [1]. In that model, opinion is quantified by a continuous variable $0<x<N$ with $N$ the opinion spectrum. Agents can interact and reach fair compromise but only if their opinion difference falls below a fixed threshold, set to unity without loss of generality [3]

$$
\left(x_{1}, x_{2}\right) \rightarrow\left(\frac{x_{1}+x_{2}}{2}, \frac{x_{1}+x_{2}}{2}\right) \quad \text { if } \quad\left|x_{1}-x_{2}\right|<1 .
$$

The probability density $P(x, t)$ of agents with opinion $x$ 
at time $t$ obeys the evolution equation [3]

$$
\begin{aligned}
\frac{\partial}{\partial t} P(x, t) & =\iint_{\left|x_{1}-x_{2}\right|<1} d x_{1} d x_{2} P\left(x_{1}, t\right) P\left(x_{2}, t\right) \\
& \times\left[\delta\left(x-\frac{x_{1}+x_{2}}{2}\right)-\delta\left(x-x_{1}\right)\right]
\end{aligned}
$$

If the restriction of the integration range is ignored, this equation describes inelastic collisions $[30-33]$. According to the interaction (17), opinion clusters are now perfectly localized (delta-functions) and in the steady-state these localized clusters are separated by distance larger than unity.

Consider the uniform initial condition: $P(x, 0)=0$ for $x<0$ or $x>N$ and $P(x, 0)=1$ for $0 \leq x \leq N$. This state is unstable with respect to perturbations that propagate from the boundary into the unstable uniform state. According to (18), a small periodic disturbance $P(x, t)-1 \propto \exp [i(k x-\omega t)]$ has the dispersion relation

$$
\omega=2 i\left[2 \frac{\sin (k / 2)}{k / 2}-\frac{\sin k}{k}-1\right] \text {. }
$$

The fastest growing mode follows from $d \omega / d k=0$ which yields $k_{\text {linear }}=2.7906$ or alternatively $L_{\text {linear }}=2.2515$. The solution to (7) is now $k_{*} \equiv k_{\text {front }}+i \lambda$ with $k_{\text {front }}=3.083750$. The decay constant $\lambda=1.294620$ characterizes the exponential decay far into the unstable state, $\phi(x) \sim \exp [-\lambda(x-v t)]$. The wavelength of perturbations at the propagating front is $L_{\text {front }}=2.037514$. The propagation velocity $v=$ $\operatorname{Im}\left[w_{*}\right] / \operatorname{Im}\left[k_{*}\right]$ is $v=0.794754$. Far behind the propagating front, that is $x \ll v t$, localized clusters form, and these clusters are separated by distance $L$. The corresponding wavenumber is $k=2.924255$ and the corresponding wavelength is

$$
L=2.1486444707 \text {. }
$$

The wavelength estimated using numerical integration results for relatively small values of $N[3], L \approx 2.155$, is reasonably close to the exact result (20).

In summary, we studied pattern formation in the bounded confidence model of opinion dynamics. Our focus was the wavelength that governs the mosaic of frozen clusters that develop, starting from a uniform state. We obtained analytically the two wavelengths that govern the pattern formation process: the wavelength of perturbations at the leading edge of the traveling wave front and the wavelength of the resulting patterns in the wake of the wave. We examined discrete and continuous versions of the bounded confidence model. In the former case, we verified the theoretical predictions using high-precision numerical measurements of the pattern wavelength and propagation velocity.

The wavelength of the patterns is irrational and since it is not commensurate with the regular lattice, the pattern formation process is hierarchical. Frozen clusters constitute the "building bocks" in this hierarchy. Integer number of clusters form quasi-periodic structures and the period of these super-patterns is an integer, too. Next, a larger number of clusters form a more intricate superpattern with a larger integer period. The numbers of clusters and the periods that characterize these superpatterns follow from continued fraction representation of the irrational wavelength governing the pattern formation process.

We observed that not all rational approximations of the wavelength necessarily correspond to a super-pattern (see figure 6). Further analysis is therefore needed to understand why certain integer fractions are realized while others are not, and more generally, to characterize the intricate structures of the super-patterns.

We acknowledge financial support from the US Department of Energy through grant DE-AC52-06NA25396 and from the US National Science Foundation through grants DMS-0806614 and DMS-1311740.

[1] G. Weisbuch, G. Deffuant, F. Amblard, and J. P. Nadal, in Interacting Agents and Continuous Opinions Dynamics, edited by G. Fandel and W. Trockel, Lecture Notes in Economics and Mathematical Systems No. 521 page 225 (Springer Verlag, Berlin, 2002).

[2] R. Hegselmann and U. Krause, J. Artif. Soc. Soc. Simul. 5, no. 3 (2002).

[3] E. Ben-Naim, P. L. Krapivsky, and S. Redner, Physica D 183, 190 (2003).

[4] E. Ben-Naim, Europhys. Lett. 69, 671 (2005).

[5] G. Weisbuch, Eur. Phys. Jour. B 38, 339 (2004).

[6] S. Fortunato, V. Latora, A. Pluchino, and A. Rapisarda, Int. Jour. Mod. Phys. C 16, 1535 (2005).

[7] J. Lorenz, Int. Jour. Mod. Phys. C 18, 1819 (2007).

[8] C. Castellano, S. Fortunato, and V. Loreto, Rev. Mod. Phys. 81, 591 (2009).

[9] B. Kozma and A. Barrat, Phys. Rev. E 77, 016102 (2008).

[10] K. Sznajd-Weron and J. Sznajd, Int. Jour. Mod. Phys. C 11, 1157 (2000).

[11] F. Slanina and H. Lavicka, Eur. Phys. Jour. B 35, 279 (2003).

[12] T. V. Martins, M. Pineda, and R. Toral, EPL 91, 48003 (2010).

[13] M. Mobilia, EPL 95, 50002 (2011).

[14] C. E. La Rocca, L. A. Braunstein, and F. Vazquez, EPL 106, 40004 (2014).

[15] R. A. Fisher, Ann. Eugenics 7, 355 (1937).

[16] A. Kolmogorov, I. Petrovsky, and N. Piscounov, Moscou Univ. Bull. Math. A 1, 1 (1937).

[17] W. van Saarloos, Phys. Rep. 386, 29 (2003).

[18] U. Ebert and W. van Saarloos, Physica D 146, 1 (2000.

[19] D. A. Kessler, Z. Ner, and L. M. Sander, Phys. Rev. E 58, 107 (1998).

[20] R. Goh, S. Mesuro, and A. Scheel, SIAM Jour. Appl. Dynam. Sys. 10, 360 (2011).

[21] M. Holzer, and A. Scheel, J. Nonl. Sci 24, 661 (2014). 
[22] G. Dee, and J.S. Langer, Phys. Rev. Lett. 50, 383 (1983).

[23] E. Brunet and B. Derrida, Phys. Rev. E 56, 2597 (1997).

[24] M. Dolnik,T. Bánsági, S. Ansari, I. Valent, and I.R. Epstein, Phys. Chem. Chem. Phys. 13, 12578 (2011).

[25] M. Wilczek, and S.V. Gurevich, Phys. Rev. E, 90, 042926 (2014).

[26] A. Scheel, arXiv:1210.4444

[27] K. Bose, T. Cox, S. Silvestri, P. Varin, M. Holzer, A. Scheel. SIURO 6, 228 (2013).

[28] M. Kotzagiannidis, J. Peterson, J. Redford, A. Scheel, Q. Wu. RIMS Kokyuroku Bessatsu B31, 79 (2012).
[29] Y. Mau, L. Haim, A. Hagberg, and E. Meron Phys. Rev. E 88, 032917 (2013).

[30] E. Ben-Naim and P. L. Krapivsky, Phys. Rev. E 61, R1 (2000).

[31] E. Ben-Naim and P. L. Krapivsky, Phys. Rev. E 73, 031109 (2006).

[32] S. Swaminathan, D. Karpeev, and I. S. Aranson, Phys. Rev. E 77, 066206 (2008).

[33] M. Romensky, V. Lobaskin, and T. Ihle, Phys. Rev. E 90, 063315 (2014). 